\begin{tabular}{cc} 
Sharif University of Technology \\
Scientia Iranica \\
SCIENTIA & Transactions B: Mechanical Engineering \\
I RAN I CA & \\
\hline
\end{tabular}

\title{
Static and dynamic path planning of humanoids using an advanced regression controller
}

\author{
P.B. Kumar*, C. Sahu, D.R. Parhi, K.K. Pandey, and A. Chhotray \\ Robotics Laboratory, Mechanical Engineering Department, National Institute of Technology, Rourkela-r769008, Odisha, India.
}

Received 24 August 2017; received in revised form 19 October 2017; accepted 8 January 2018

\author{
KEYWORDS \\ Humanoid NAO; \\ Regression controller; \\ Path planning and \\ navigation; \\ Petri-net model; \\ Dynamic path \\ planning; \\ V-REP.
}

\begin{abstract}
With the ability to mimic human behaviour, humanoid robots have become a topic of major interest among research fellows dealing with robotic investigation. The current work is focused on the design of a novel navigation controller based on the logic of the regression analysis to be used in the path planning and navigation of humanoid robots. The current investigation focuses on static and dynamic path planning of humanoid NAOs. The static path planning represents a single NAO navigating through random static obstacles. The dynamic path planning represents multiple humanoid NAOs navigating through random static obstacles and acting as dynamic obstacles for each other. A Petri-net controller is designed to avoid the collision among multiple NAOs in dynamic path planning. To reduce path length and time travel and provide the shortest possible path, an advanced regression controller is implemented in the NAOs in both simulation and experimental environments. Finally, a comparison has been performed between the simulation and experimental results, and good agreement is observed between both of the results with a minimal percentage of error. The proposed navigation controller is also tested against other existing navigational technologies to validate better efficiency.
\end{abstract}

(C) 2019 Sharif University of Technology. All rights reserved.

\section{Introduction}

With the development of science and technology, robots have become an integral part of human life. As its basic definition states, a robot is a device or equipment that can reduce and sometimes replace human efforts. Out of all the categories of robots, such as wheeled mobile robots, biped robots, etc., humanoid robots have gained immense popularity in almost all sectors of the present-day technology. Their human-mimicking behaviour and capability to use the same workplace as used by humans make the humanoid form more

\footnotetext{
*. Corresponding author.

E-mail addresses: p.biplabkumar@gmail.com (P.B. Kumar); mechchinu@gmail.com (C.Sahu);dayalparhi@yahoo.com (D.R. Parhi); kknitrkl@yahoo.in (K.K. Pandey); chhotrayanimesh@gmail.com (A.Chhotray)
}

advantageous than other forms. A humanoid robot encounters a general problem of path planning and obstacle avoidance in workplace. If a humanoid robot is intended to be used in a cluttered environment, then it should be able to detect potential obstacles present in its path and reach the destination without colliding with the obstacles. Hence, robot path planning and navigation is considered as one of the most challenging areas of research. Path planning problem can be categorised as static path planning and dynamic path planning. This categorisation is based on the presence of obstacles types. As the name suggests, robot has to deal with static obstacles in static path planning and with dynamic obstacles in dynamic path planning. Dynamic path planning is much more critical than static path planning. To enable a robot to work in a complex environment, it has to deal with moving objects and human beings.

Path planning approaches are categorised as clas- 
sical techniques dealing with computational intelligence. The path planning and navigation of humanoid robots is very recent and modern, as compared to path planning of wheeled mobile robots. In the current work, regression analysis is chosen as the path planning approach to a humanoid NAO. Regression analysis is a classical technique and provides more convergent results. Several researchers have discussed regression analysis and the path planning approach of robots. Some of them can be discussed herein. Atkinson [1] applied regression analysis to three specific problems in which the least-square estimation and basic regression were in sharp contradiction. He concluded that the efficiency of the regression analysis is largely dependent on the linear model that represents the problem. Asano et al. [2] discussed a method for constructing any specific design by the use of disjoint polygons. Takahashi and Schilling [3] designed an algorithm to generate a collision-free path in a rectangular workspace cluttered with polygon-shaped obstacles. Hwang and Ahuja [4] presented a method of multi-level path planning. The idea behind the use of a multi-level path planning is to ensure higher accuracy of results. Lazaro et al. [5] used the sensory data to be implemented in a pathplanning algorithm. By collecting sensory data, the information regarding the presence of any obstacle can be confirmed. Bai and Low [6] proposed a method for linking the body trajectory planning to the terrain evaluation to make the path-planning problem easy and less time consuming. They constructed a potential field based on the terrain evaluation for a graphical search method. Lee and Bien [7] encountered the problem of path planning for a quadruped robot by means of an artificial thermal field. In their analysis, parameters such as obstacle avoidance, goal seeking and stable gait trajectory were considered. Minguez and Montano [8] proposed a divide-and-conquer strategy for path planning. They performed the path planning by the use of nearness diagram in troubleshoot scenarios. Benamati et al. [9] introduced a flat potential field approach to the navigation of mobile robots in a static environment. Liang et al. [10] studied a non-holonomic path planning method by considering both the constraints available in the path and optimisation of the path. Papadopoulos et al. [11] developed a nonholonomic path planning technique for mobile manipulators. Their approach was based on polynomial calculations. Masehian and Sedighizadeh [12] contributed almost 35 years of development in the path planning approach in a review form based on concept of Zero Moment Point (ZMP). Qi et al. [13] redesigned the previously followed potential field approach and named it an artificial potential field method for path planning of mobile robots. Jolly et al. [14] introduced the Bezier curve technique in path planning. They applied the technique to a multi-agent robot soccer system. Keshmiri and Payandeh [15] designed a regression route for a multi-robot and multirecharging station problem. Their main objective was to enable robots to use the nearest recharging station without sticking to a particular recharging station and avoiding disturbance to each other. Shi and Zhao [16] improved the potential field method by overcoming the problem of trapping at local minima and reaching the destination safely. Singh et al. [17-19] discussed the use of computational intelligence for smooth and collisionfree path generation for wheeled mobile robots. Razzazi and Sepahvand [20] proposed an idea regarding time complexity of two disjoint simple paths. Their study is primarily applicable to path planning of mobile robots. Mohammadi et al. [21] proposed a wearable exoskeleton model for a disabled human arm to facilitate various operations. They considered kinematic constraint data to design their model. Kala et al. [22] applied a hierarchical evolutionary algorithm to both static and dynamic path planning of a robot. They used two hierarchies, i.e., coarse and finer hierarchies, for the path planning to enable the robot to cross both static as well as dynamic obstacles. Kashmiri and Payandeh [23] proposed a kernel regression technique for the navigation of a robotic agent in both static and dynamic environments. Sheng et al. [24] further contributed to the artificial potential field approach by adding a feature of path planning of a virtual human. Hong et al. [25] attempted dynamic path planning of a mobile robot by the use of the artificial potential field approach. Mohanty et al. [26-28] developed several navigational techniques for path planning of mobile robots using artificial intelligence. They discussed modification of controlling parameters of basic intelligent algorithms for performance improvement. Pham and Parhi [29] discussed the navigation of multiple mobile robots using a neural network and a Petrinet model. Chen and Ko [30] designed a visionbased approach to the self-localization of soccer playing robots. They altered the intensity of the lighting system to test the effectiveness of the design. Korayem et al. [31] proposed a graphical user interface for a cable suspended robot using LabVIEW software. They considered both kinematic and control parameters in their analysis. Sayyaadi and Babaee [32] designed a control scheme for a mobile manipulator to perform object transportation tasks. They tested the manipulator for approaching and grasping objects in a simulation environment. Ohki et al. [33] proposed a local path planner based on the distance time transform method for mobile robot navigation in dynamic environments. They applied the technique based on the current and future possible positions of dynamic obstacle so that the robot can reach its destination without colliding with the obstacles. Li et al. [34] applied the regression technique to the path planning of mobile robot considering prior information about environmental con- 
ditions. Hong and Park [35] designed a navigation strategy for mobile robots based on a turning point search. They considered an obstacle avoidance strategy and path optimization in their technique. Keshmiri and Payandeh [36] applied regression technique to a multi-robot and multi-recharging optimisation problem. Tingbin and Qisong [37] performed robot path planning using a stable and mature artificial potential field approach. Clever and Mombaur [38] introduced an inverse optimal control to transfer motion from humans to humanoids. They prepared a 3D template model to extract motion constraints from a human walking and used the extracted parameters in humanoid walking. Mirjalili et al. [39] developed an online path planning approach to SURENA III humanoid robot based on control schemes.

Based on the extensive discussion on the available literatures, it has been observed that many researchers have tried path planning of wheeled mobile robots, yet path planning of humanoid robots is very rare to find. Although path planning in static environments is available, work on dynamic environments has not been reported significantly. A humanoid is subjected to both static and dynamic environments while working in a real life environment. Based on the shortcomings of the available literatures, the current work is aimed at static and dynamic path planning of humanoid NAO using a regression navigational controller. The primary objective of the work is to design a novel navigation controller that can navigate single and multiple humanoids in complex environments. Since humanoid navigation is relatively new in its own kind, the present work needs to be treated as a pioneering art in the field of humanoid robotics.

\section{Humanoid NAO25}

Several humanoid robots were developed in the past with varying features. In 2004, Aldebaran group of France came up with NAO, which is a small-sized and programmable humanoid robot. Starting from the introduction in market to replacing quadruped robot AIBO of Sony in 2007, NAO has been modified through several versions [40]. Humanoid NAO, version V3.3, has been used in the current analysis. The current version of NAO has 25 degrees of freedom. The performance of NAO is largely dependent on its sensor network. The sensor network of NAO includes eight pressure sensors, nine tactile sensors, one inertial board, two infrared receivers and emitters, two sonar range finders, four microphones, and two cameras. NAO has a weight of about $5 \mathrm{~kg}$ and a height of about $58 \mathrm{~cm}$. The efficient sensory network of NAO enables it to gather information about environmental conditions and act accordingly. Since the path-planning problem includes sensory data as the input parameters,

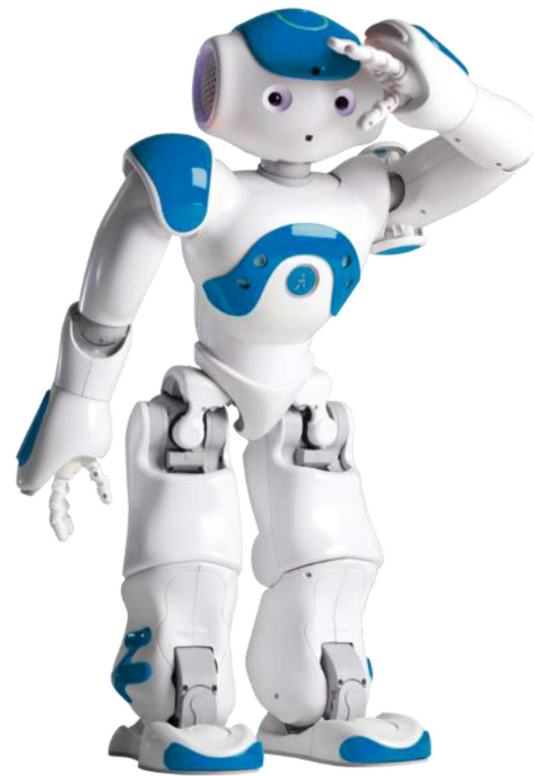

Figure 1. A typical humanoid NAO.

humanoid NAO can be very handy in path optimisation problem. Figure 1 represents a humanoid NAO.

\section{Outline of basic regression analysis}

The path-planning problem can be tackled by the use of either classical approaches or computational intelligence approaches. These two approaches can also be stated as deterministic and non-deterministic approaches, respectively. Classical approaches are popular due to their deterministic nature, and the results converge based on their ability within limited time. Regression analysis is one of the popular classical techniques used for optimisation problems. Considering that the path planning of the humanoid robot is very new in its own kind, Regression Analysis (RA) is chosen in the current work as the method to be implemented in the humanoid NAO to enable it to reach the goal position without colliding with the obstacles present in the path. Regression analysis is also a statistical approach that can relate some variables used in a system. It can be used to formulate a relation among dependent and independent variables. If the independent variable is kept fixed in a system, then the change in the dependent variables can be observed with respect to the independent variable. Regression technique can also be called a data forecasting technique. In basic regression, the dependent variables are not exactly linear to the independent variables; rather, they are linear to some parameters concerned with the independent variables. A simple equation of regression can be represented as follows:

$$
y_{i}=\beta_{1}+\beta_{2} x_{i}+e_{r} \quad i=1,2,3, \cdots, n .
$$

In Eq. (1), $y_{i}$ is dependent upon $x_{i}$ with $\beta_{1}$ and $\beta_{2}$ as 


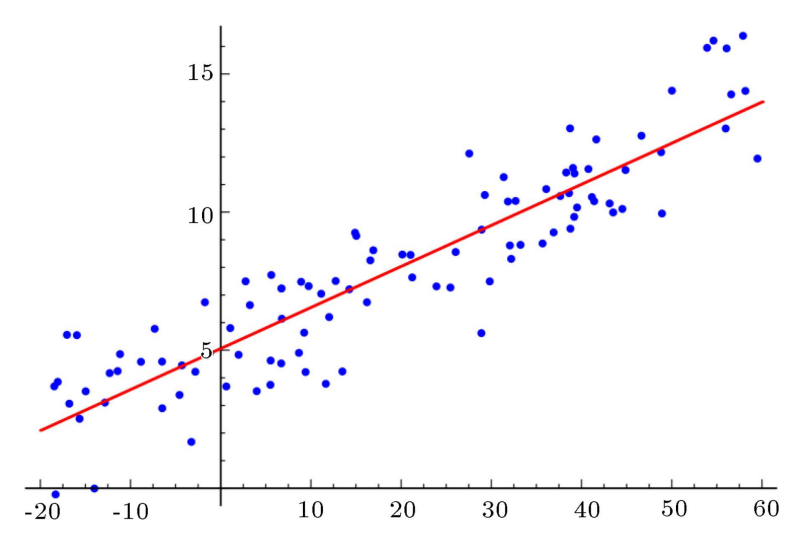

Figure 2. Description of a linear regression.

the parameters relating the dependent parameters to the independent ones. Factor $e_{r}$ is a term associated with any error factor. The most significant feature of regression analysis is the accumulation of scattered data in the equation form to a straight line so that the results can converge to a common solution. Figure 2 represents a basic regression data accumulation.

In Figure 2, the independent parameter is represented in the horizontal axis, and a dependent parameter in the vertical axis. The values from -20 to 60 in the $x$-axis represent ranges of independent variable, and the values from 0 to 15 in the $y$-axis represent ranges of the dependent variable with respect to the independent variable. For each value on the $x$-axis, there are corresponding data on the $y$-axis. Finally, all the scattered data are brought together in the equation form to a straight line, representing the solution to the problem.

\section{Control architecture of humanoid path planning using regression analysis}

The primary objective of any path planning and navigation problem is to avoid the obstacles present in the path and reach the target position in the shortest route consuming less possible time. The optimisation of the navigational problem depends on the optimisation of the parameters that influence the path-planning problem significantly. In the navigation of a humanoid NAO, four parameters are considered. Those are Front Obstacle Distance (FOD), Left Obstacle Distance (LOD), Right Obstacle Distance (ROD), and Turning Angle (TA). Out of the above four parameters, FOD, LOD, and ROD act as the input parameters, and TA acts as the output parameter. The input parameters are based on the sensory data fed to the controller, and the output parameter is the result of computation done by the controller. To have a better understanding regarding these navigational parameters, Figure 3 can be considered. In Figure $3, X_{1}, X_{2}$, and $X_{3}$ represent LOD, ROD, and FOD, respectively.

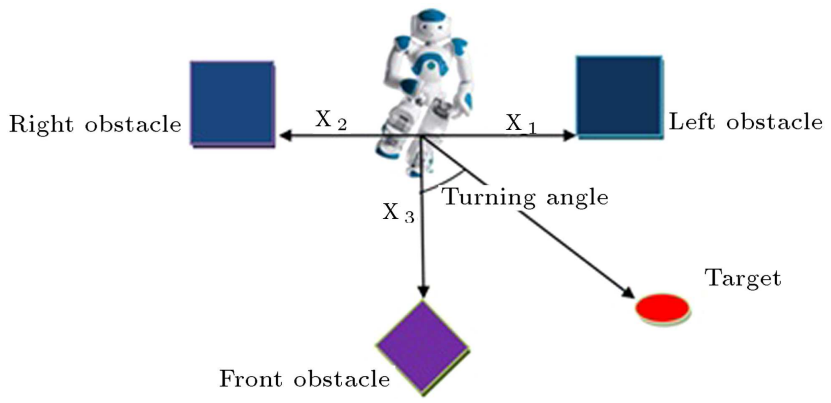

Figure 3. Navigational parameters of the humanoid NAO.

The input parameters are the distances of the obstacles present near the humanoid NAO. NAO is equipped with different types of sensors. The sensors attached with the NAO record the presence of obstacles in the suitable range and feed the data to the microcontroller of the humanoid.

Depending upon the feeding of environmental conditions to a robot, the path planning can be categorised as global path planning and local path planning. In global path planning, the robot has prior information regarding the environmental conditions; in local path planning, the robot is unaware of the environmental condition. In the current work, the robot is aware of the start position and goal position. The primary objective of path planning is that the robot is always headed towards the goal position. Unless the humanoid encounters any obstacle in the path, it moves without activation of the controller towards the specified goal position. Once the sensors detect any obstacle, the path planning algorithm works as per the designed method. In regression analysis, some training data are fed to the system so that the controller can be efficient in choosing any possible case of the obstacle avoidance problem. Based on the environmental conditions provided to the humanoid NAO, some training patterns of about 1000 data are fed to the controller. Table 1 illustrates some of the training pattern data.

In Table 1, the input parameters of FOD, LOD, and ROD are measured in centimetres of distance of robot from the obstacle, and the output parameter TA is measured as the degrees of turning. By taking one example of the training data, the navigational parameters can be understood. By taking the 54th data from Table 1, it is observed that FOD, LOD, and ROD are $42 \mathrm{~cm}, 32 \mathrm{~cm}$, and $48 \mathrm{~cm}$, respectively. A threshold distance is set for the sensors of the humanoid to detect the obstacles. In the current study, the threshold distance is set to $30 \mathrm{~cm}$. In the above example, the value of left obstacle distance is minimum which signifies that the robot is near to an obstacle, which is present in the left side. To avoid the obstacle, the robot must take a right turn, so the 
Table 1. Examples of some training pattern data for RA navigation controller.

\begin{tabular}{|c|c|c|c|c|c|c|c|c|c|}
\hline $\begin{array}{l}\text { Sl. } \\
\text { no }\end{array}$ & $\begin{array}{l}\text { FOD } \\
(\mathrm{cm})\end{array}$ & $\begin{array}{l}\text { LOD } \\
(\mathrm{cm})\end{array}$ & $\begin{array}{c}\text { ROD } \\
(\mathrm{cm})\end{array}$ & $\begin{array}{c}\text { TA } \\
\text { (degree) }\end{array}$ & $\begin{array}{l}\text { Sl. } \\
\text { no }\end{array}$ & $\begin{array}{l}\text { FOD } \\
(\mathrm{cm})\end{array}$ & $\begin{array}{l}\text { LOD } \\
(\mathrm{cm})\end{array}$ & $\begin{array}{l}\text { ROD } \\
(\mathrm{cm})\end{array}$ & $\begin{array}{c}\text { TA } \\
\text { (degree) }\end{array}$ \\
\hline 1 & 60 & 30 & 50 & 0 & 31 & 50 & 60 & 30 & -14 \\
\hline 2 & 30 & 40 & 60 & 10 & 32 & 60 & 30 & 40 & 16 \\
\hline 3 & 35 & 70 & 50 & 20 & 33 & 50 & 35 & 70 & 12 \\
\hline 4 & 30 & 55 & 30 & -15 & 34 & 30 & 30 & 55 & 15 \\
\hline 5 & 70 & 30 & 40 & 0 & 35 & 40 & 70 & 30 & -17 \\
\hline 6 & 40 & 40 & 30 & -25 & 36 & 30 & 40 & 40 & -25 \\
\hline 7 & 45 & 35 & 50 & 20 & 37 & 50 & 45 & 35 & -18 \\
\hline 8 & 35 & 55 & 40 & -15 & 38 & 40 & 35 & 55 & 20 \\
\hline 9 & 80 & 45 & 55 & 0 & 39 & 55 & 80 & 45 & -22 \\
\hline 10 & 38 & 59 & 42 & 10 & 40 & 42 & 38 & 59 & 18 \\
\hline 11 & 36 & 39 & 48 & 12 & 41 & 48 & 36 & 39 & 15 \\
\hline 12 & 65 & 46 & 58 & 0 & 42 & 58 & 65 & 46 & -11 \\
\hline 13 & 46 & 66 & 52 & -19 & 43 & 52 & 46 & 66 & 14 \\
\hline 14 & 51 & 65 & 33 & 0 & 44 & 33 & 51 & 65 & -12 \\
\hline 15 & 41 & 53 & 62 & 16 & 45 & 62 & 41 & 53 & 18 \\
\hline 16 & 32 & 61 & 55 & -18 & 46 & 55 & 32 & 61 & 16 \\
\hline 17 & 48 & 85 & 65 & 0 & 47 & 65 & 48 & 85 & 12 \\
\hline 18 & 34 & 38 & 42 & 26 & 48 & 42 & 34 & 38 & 15 \\
\hline 19 & 34 & 42 & 38 & -25 & 49 & 38 & 34 & 42 & 16 \\
\hline 20 & 38 & 42 & 34 & -24 & 50 & 34 & 38 & 42 & -18 \\
\hline 21 & 39 & 55 & 45 & -18 & 51 & 45 & 39 & 55 & -21 \\
\hline 22 & 36 & 61 & 71 & 14 & 52 & 71 & 36 & 61 & 0 \\
\hline 23 & 53 & 36 & 38 & 0 & 53 & 38 & 53 & 36 & -16 \\
\hline 24 & 32 & 48 & 42 & -20 & 54 & 42 & 32 & 48 & 18 \\
\hline 25 & 35 & 45 & 52 & 18 & 55 & 52 & 35 & 45 & 20 \\
\hline 26 & 45 & 53 & 62 & 19 & 56 & 62 & 45 & 53 & 25 \\
\hline 27 & 70 & 58 & 36 & 0 & 57 & 36 & 70 & 58 & 12 \\
\hline 28 & 42 & 45 & 48 & 22 & 58 & 48 & 42 & 45 & 28 \\
\hline 29 & 45 & 56 & 49 & -15 & 59 & 49 & 45 & 56 & 19 \\
\hline 30 & 35 & 65 & 20 & -18 & 60 & 20 & 35 & 65 & -26 \\
\hline
\end{tabular}

turning angle is set to 18 degrees. It can be noticed that, in Table 1 , a negative sign is attached with some turning angles. It is not to be confused that the negative sign represents any type of a negative turning angle. It only symbolises that the robot is taking a left turn. A specific set of sign conventions is chosen for the humanoid robot to encounter the path-planning problem. The sign conventions can be understood perfectly through Figure 4.
It can be noticed from Figure 4 that a positive turning angle represents a right turn for the humanoid. A negative turning angle represents a left turn, and a zero turning angle represents that the humanoid is moving in a straight path without any turning.

After deciding the specific sign conventions and training patterns for the regression navigational controller, the training data are fed to the regression toolbox of Minitab software. The primary aim of using 


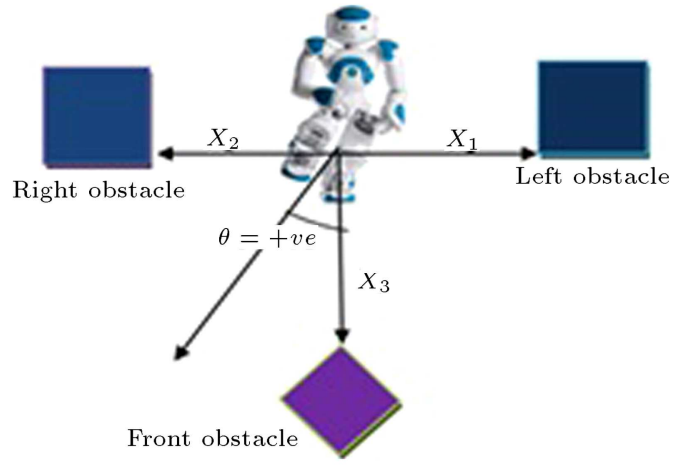

(a)

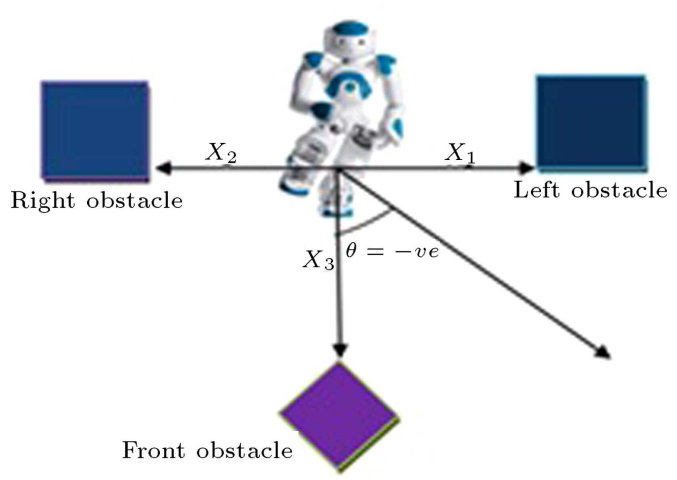

(b)

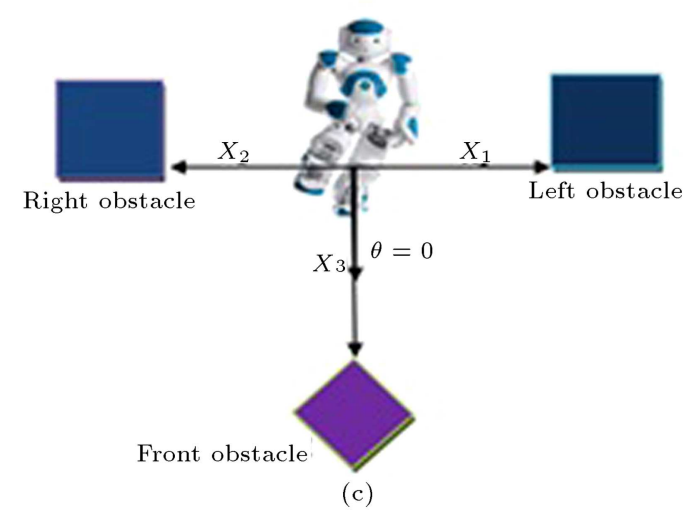

Figure 4. Sign conventions used in RA navigation controller.

the Minitab software is to formulate a solution in an equation format that can be used in the output of the system. The Minitab software generated the equation that can be represented as follows:

$$
\begin{aligned}
K_{4}= & -23.0228-0.006183 K_{1}-0.28508 K_{2} \\
& +0.78636 K_{3},
\end{aligned}
$$

where $K_{1}$ is front obstacle distance, $K_{2}$ is left obstacle distance, $K_{3}$ is right obstacle distance, and $K_{4}$ is turning angle.

Based on Eq. (2), the regression navigational controller is designed and implemented in the humanoid NAO. The path-planning problem of the humanoid NAO is considered as the global path-planning problem in which the humanoid has the prior information on the start and goal positions. The humanoid is always headed towards the goal position obeying the goal following behaviour. Unless there is an obstacle present in the path, the humanoid moves towards the goal without using any control algorithm. Once any obstacle is detected by the sensory network of the humanoid, the input parameters are calculated as described earlier. By considering the input parameters, the navigation controller analyses the scenario and generates the required turning angle to overcome the obstacle. Following the obstacle avoidance, again the humanoid obeys the goal following behaviour and moves towards the target. The values of the constants as generated by the Minitab software, i.e., -23.0228 , $-0.006183,-0.28508$, and 0.786367 , are dependent upon the training pattern data. In the current analysis, a total of 1000 data are used. These values can also be more converged by using some more training pattern data, refining the results up to some extent.

A humanoid NAO follows various types of reactive behaviours such as obstacle avoidance behaviour, goal seeking behaviour, and barrier following behaviour. These three reactive behaviours are very significant in the context of path planning problem formulation. The obstacle avoidance behaviour deals with the setting of turning angle to avoid the obstacle if present in the path as sensed by the sensory network of the humanoid. The goal seeking behaviour states that the humanoid should always head towards the goal if there is the absence of any obstacle in the path. In the absence of any obstacle, the turning angle of the robot is set to zero and is directed towards the target. While the obstacle avoidance and goal seeking behaviours are compulsory behaviours for the navigation of the humanoid, the barrier following behaviour is a complimentary one. The third behaviour states that if a long series of obstacles or barriers are present in the path of the humanoid and the target is close to the end of the long barrier, then the robot just follows the barrier without activation of the navigation controller. In this scenario, the microcontroller is not activated; low amount of energy is utilised in the navigation, which in turn increases the efficacy of the algorithm. Table 2 summarises the reactive behaviours followed by a humanoid robot during path planning and navigation.

Figure 5 represents different reactive behaviours followed in the humanoid navigation. These reactive behaviours are based on the above-discussed behaviours.

While designing the control algorithm for the navigation of the humanoid robots, all the logics of regression analysis and different reactive behaviours are considered into account. Figure 6 represents the pseudo code for the proposed RA navigation controller, and 
Table 2. Reactive behaviours followed in path planning of a humanoid NAO.

\begin{tabular}{cll}
\hline $\begin{array}{c}\text { Types of reactive } \\
\text { behaviors }\end{array}$ & \multicolumn{1}{c}{$\begin{array}{c}\text { Explanation of the } \\
\text { reactive behaviors }\end{array}$} & Activity of the robot \\
\hline Obstacle Avoidance (OA) & $\begin{array}{l}\text { To avoid the obstacles present } \\
\text { in the path when sensed by the } \\
\text { sensors of a robot. }\end{array}$ & $\begin{array}{l}\text { Turning angle is set } \\
\text { accordingly to avoid } \\
\text { the obstacles. }\end{array}$ \\
Target Seeking (TS) & $\begin{array}{l}\text { To seek the target when there } \\
\text { are no obstacles present in } \\
\text { the path. }\end{array}$ & $\begin{array}{l}\text { Turning angle is adjusted } \\
\text { accordingly to reach the } \\
\text { goal position. }\end{array}$ \\
& $\begin{array}{l}\text { To follow a barrier when searching } \\
\text { for a target if a series of obstacles } \\
\text { are present near the robot. }\end{array}$ & $\begin{array}{l}\text { Robot moves in parallel to } \\
\text { the barrier keeping a fixed } \\
\text { turning angle. }\end{array}$ \\
\hline
\end{tabular}

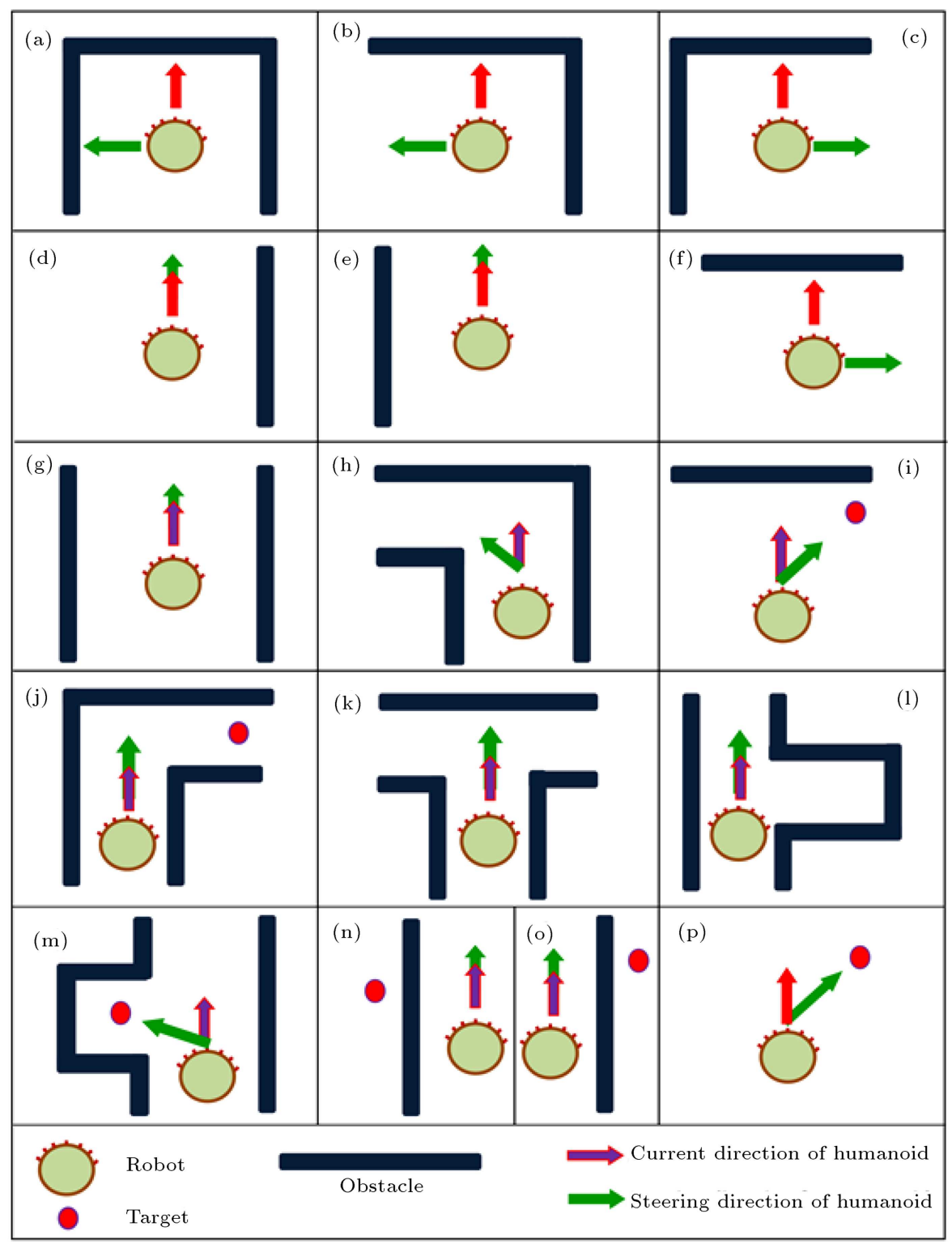

Figure 5. Reactive behaviours of RA navigation controller. 


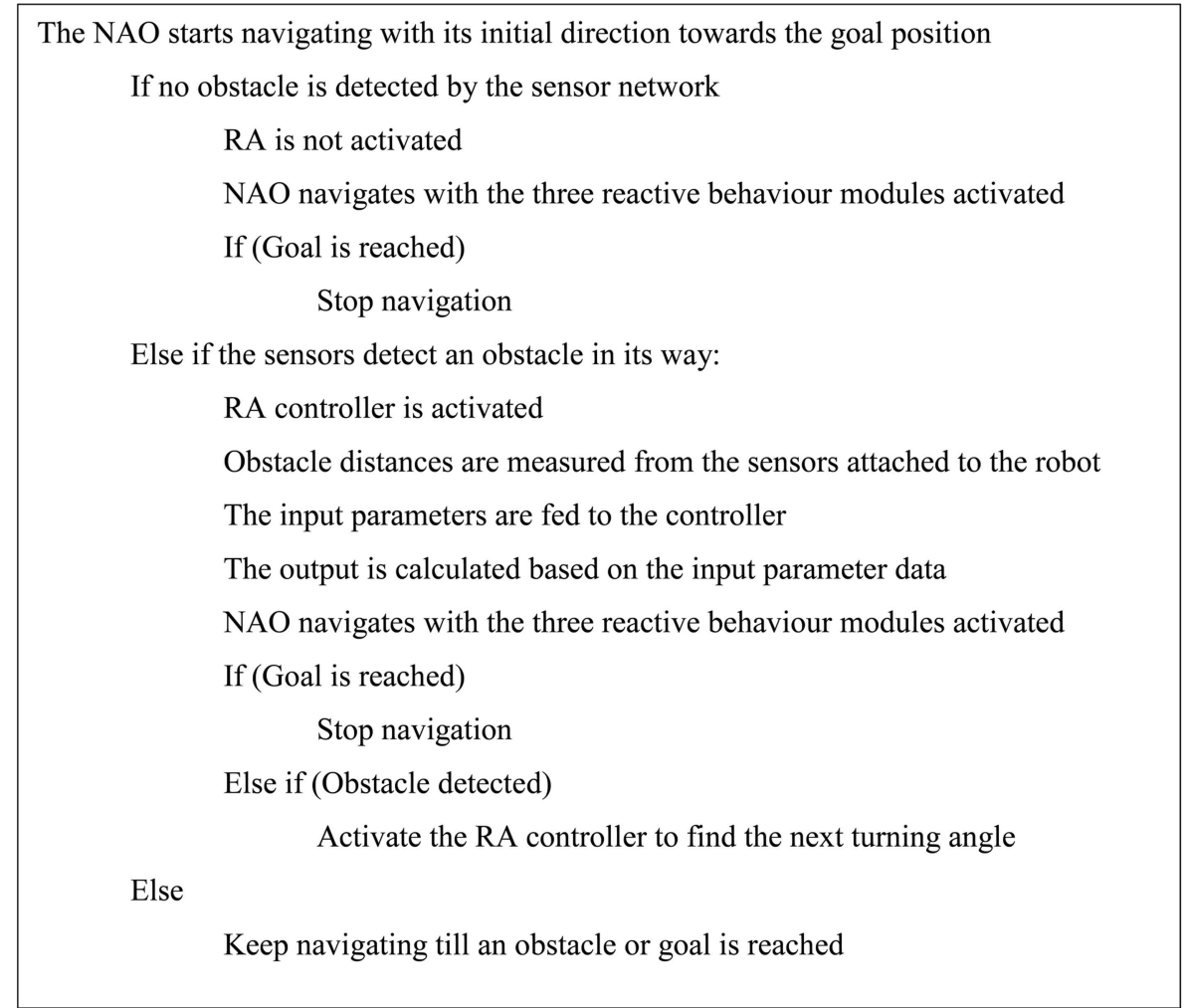

Figure 6. Pseudo code for the proposed RA navigation controller.

Figure 7 represents the flowchart for the control scheme of the proposed algorithm.

\section{Design of a Petri-net controller for avoiding inter-collision among multiple humanoids}

In the current investigation, both static and dynamic path planning problems of humanoid NAO are dealt with. In the static path-planning problem, intercollision is not encountered since only a single humanoid is navigated through some random obstacles. In the dynamic path-planning problem, multiple humanoid NAOs are navigated through random static obstacles. As all the humanoids navigate simultaneously in a common environment, it is quite evident that there may be a problem of inter-collision among themselves. To avoid the inter-collision among the humanoids, a Petri-net controller has been designed.

Peterson [41] thoroughly discussed the development of a Petri-net controller used to deal with a dynamic environment. A Petri-net controller is required to prevent the inter-collision between two dynamic obstacles. In the current investigation, dynamic path planning is also considered along with the static path planning approach. When multiple humanoid NAOs navigate in a common environment or platform, they act as dynamic obstacles for each other. The basic structure and function of a Petri-net controller as used in the current work can be discussed as follows. Figure 8 represents the structure of a Petri-net controller used in the current problem.

The position of a robot is represented as a circle, symbolising an event. The bar symbol represents transition from one state to another. The red elliptical symbol represents the current position of the robot or the token location. In our navigational problem, three humanoid NAOs navigate simultaneously in a common environment. Hence, two humanoids always act as dynamic obstacles for the first one. In the developed Petri-net model, six positions are represented. In the initial stage, it is assumed that all the humanoids are unaware of each other's location in the environment and are standing in random positions. This is the start of their journey, and the token is at Position 1. Position 1 also signifies that all the humanoids are waiting for any command to execute and start their journey to their respective targets. The second position in the model represents tracing each other's location by the sensory network. The idea behind tracing each other is to avoid any obstacle if present in the path. While Position 2 represents the detection of static obstacles, the next position, Position 3, comes to picture when there is detection of any dynamic obstacle. When two humanoids are acting as a dynamic obstacle to each other (Position 3), they have to set a priority regarding which humanoid to move further 


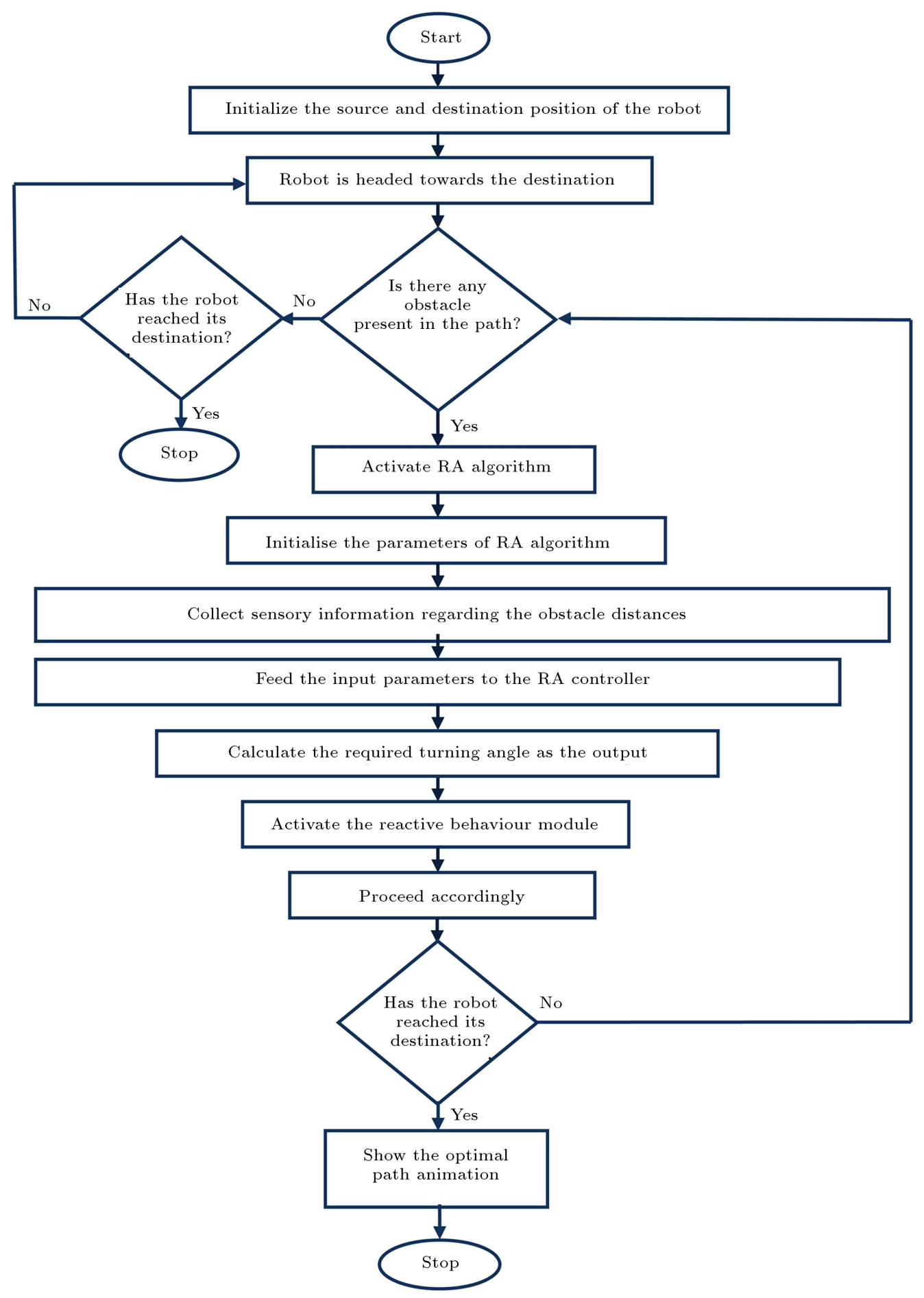

Figure 7. Flowchart of the proposed RA algorithm for navigation of humanoids.

and which one to wait until the other one moves. As per a general rule, the humanoid with minimum distance to reach the target is given more priority than the one with more distance to reach the goal. The robot with less priority would stop acting as a static obstacle until the robot with more priority leaves for forward motion. Position 4 represents this negotiating situation between two humanoids regarding setting of the priority. After encountering a negotiating situation such as Position 3, the next task is to check any further conflicting situations. In the absence of any further negotiating situations, the humanoid would move forward which is represented as Position 5 in the model. The last position, Position 6, is defined as a waiting condition. If any humanoid encounters a situation where two other humanoids are already 


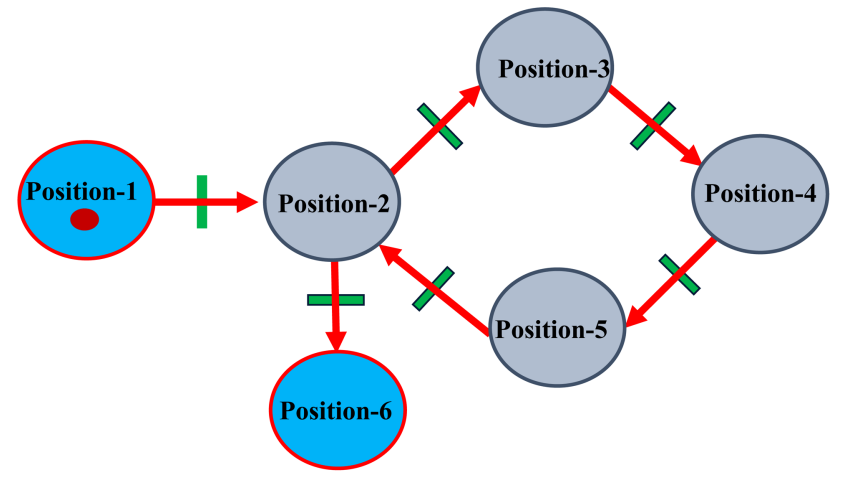

Figure 8. Developed Petri-net controller used in the navigational approach.

in a negotiating mode, it has to stop there acting as a static obstacle by giving them a higher priority. After the two negotiating humanoids move forward, the one in Position 6 would take Position 2 and proceed towards its respective target. Considering all these positions in the developed model, multiple humanoids can navigate in a common platform without encountering any collision with each other and reach their respective goals safely. The design of the proposed Petri-net model works in a simpler way, which in turn optimises the path travelled and reduces the time taken to reach the target.

\section{Navigation of a single humanoid NAO in a complex environment}

After the design of the control architecture for the path planning of the humanoid robots and the Petri-net controller for avoiding inter-collision among multiple humanoids, the proposed navigation controller was tested for both simulated and experimental environments. It is to be noted that the Petri-net controller is required when multiple humanoids navigate simultaneously in a common platform. For the navigation of a single humanoid NAO, it is not required. The purpose of this section is to check the regression navigational controller for a simulation as well as experimental platform. Finally, following the execution of the controller in both of the platforms, a comparison is aimed for the validation between the simulated and experimental results.

\subsection{Simulation and experiment in the static environment}

Several simulation software products have been developed over the past years. In the current work, V-REP is chosen as the simulation software, because it is an easy and suitable software product for humanoid navigation. V-REP follows the programming language LUA based on the ANSI C language. Specific unique properties, such as collision detection, better motion planning, and calculation of the shortest path, make V-REP a more potential candidate than other software products. To analyse the effectiveness of regression navigational controller, a static environment has been created in the $\mathrm{V}$-REP software. It has to be kept in mind that the performance of the navigation controller must be based on the reactive behaviours such as obstacle avoidance, goal following, and barrier following. The environment size was chosen as $200 \times 250$ units with five numbers of static obstacles. By considering the reactive behaviours and logic of regression analysis, a program has been written and implemented in the NAO humanoid. After implementing necessary rules and regulations, obstacle avoidance and goal following behaviours were tested. The main objective of navigational analysis is to observe the shortest path calculation and time taken to reach the desired target. Figure 9 represents the simulation results as obtained from the V-REP software.

It can be observed from Figure 9(a) that, initially, the NAO was set at a source point, and a specific destination point was provided. The two blue boxes represent the source and target points. Five obstacles were set at random positions. It was observed that NAO was able to avoid all the obstacles if present in the path and reach the desired target safely by using the proposed regression navigation controller. During the journey from Figure $9(\mathrm{a})$ to $9(\mathrm{~g})$, it can be observed that the humanoid has followed the shortest possible path. The distance covered by the humanoid to reach the destination and the time consumed to reach the target were noted from the V-REP simulation window itself and recorded.

To validate the effectiveness of the proposed regression navigational controller, it is important to repeat the simulation results in an actual environment. By creating an exactly similar environment under laboratory setup, the simulation results can be compared for practical implementation. To maintain the same environment size, the actual platform to conduct the experiment was chosen as $200 \times 250$ centimetres. Five static obstacles were selected at the same positions as those of the simulation environment. Initial and final points of the experiment were established. By using the logic of the reactive behaviours and regression analysis, a program was written and implemented in the humanoid NAO. In the actual environment, the NAO was operated by a Wi-Fi control. After the environment was set up, the navigation of NAO was observed and analysed. Figure 10 represents the actual experiment performed in our laboratory.

The two blue boxes represent the source and target positions in Figure 10(a). Five static obstacles as represented by white boxes were set at the exact similar positions as were in case of simulation. Following the establishment of the environment, the NAO was 


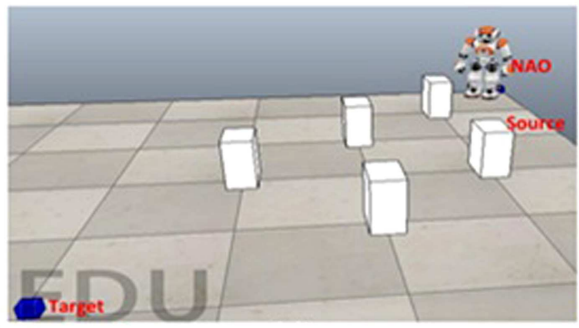

(a)

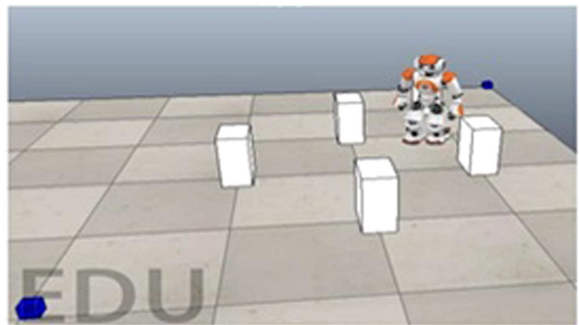

(c)

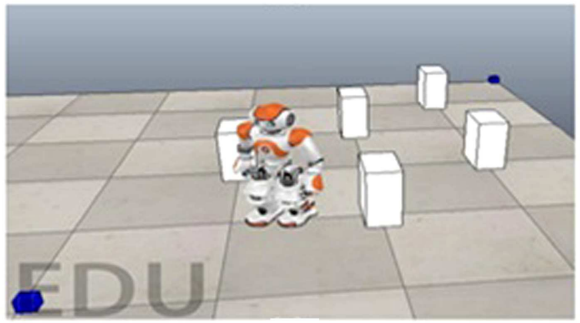

(e)

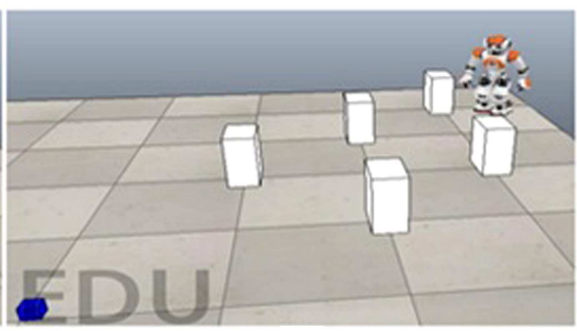

(b)

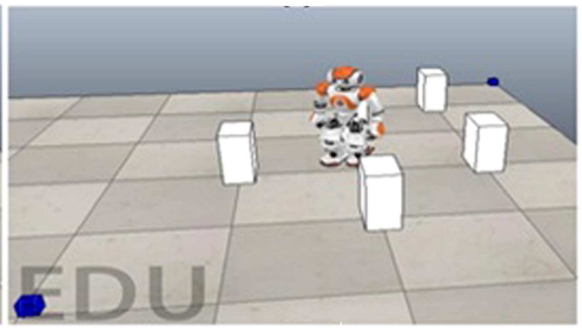

(d)

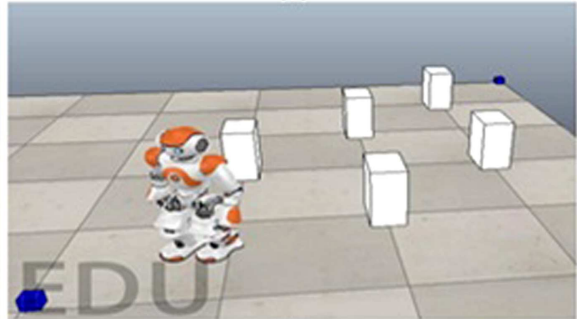

(f)

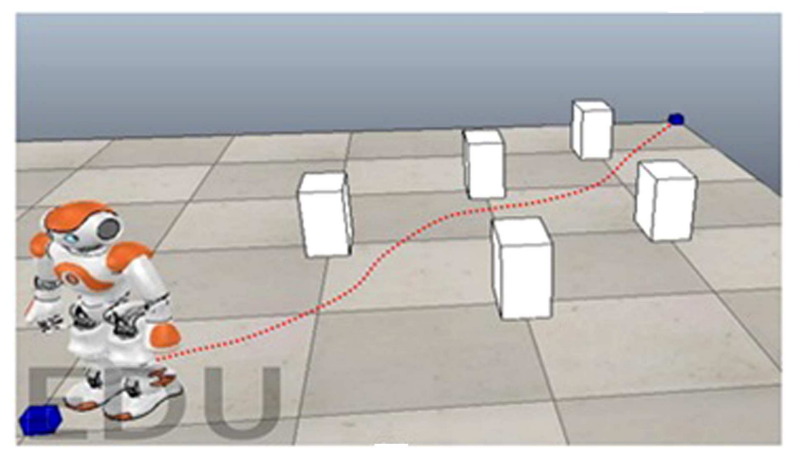

(g)

Figure 9. Simulation result for the navigation of a single NAO using RA navigation controller.

started for its navigation. It was observed that the humanoid NAO was able to avoid all the obstacles that were present in the path and reach the desired target safely. It can be observed from Figure 10(a) to $10(\mathrm{f})$ that the humanoid has followed the shortest possible route. In the actual environment, the path length from source to target as travelled by the humanoid was measured by using a measuring tape, and a stopwatch measured the time taken to reach the target. The path length and time taken were noted and recorded for the comparison between the simulated and experimental results. As stated earlier, the effectiveness of the proposed navigation controller can only be checked by the proper comparison between the simulated and experimental results regarding the navigational parameters, which are the path length and time taken. Tables 3 and 4 represent the comparison between the simulated and experimental results for path length and time taken, respectively. It can be noted that quite a large number of experiments were performed for the navigational control of humanoid NAO using regression analysis, and only a few have been analysed herein.

From Tables 3 and 4, it can be noticed that the navigational parameters for the experiments always show higher values than the simulation results. The simulation results are ideal where there are no errors such as loss of data transmission, effects of friction, etc. When the humanoid navigates in a practical environment, it is influenced by several external factors 

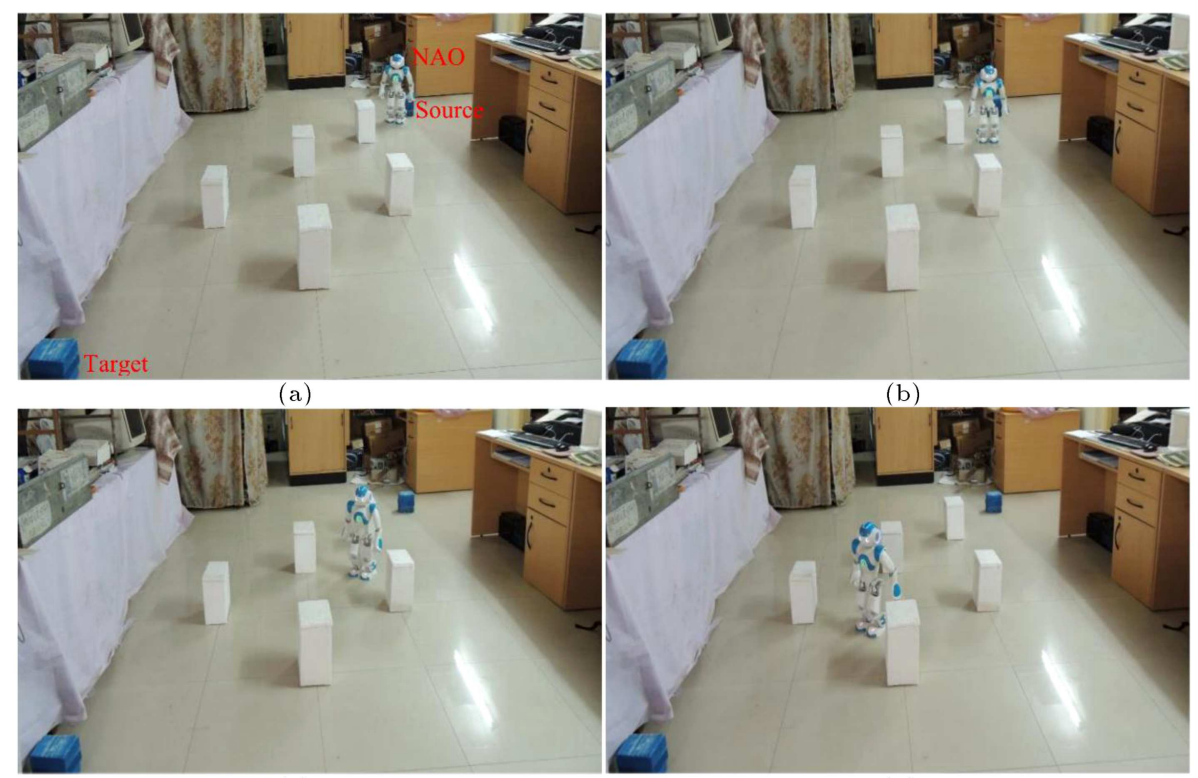

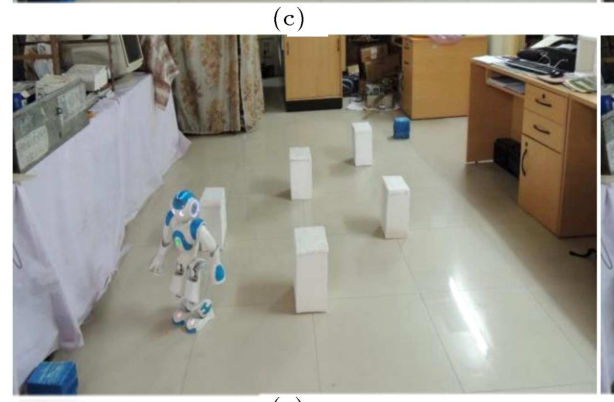

(e)

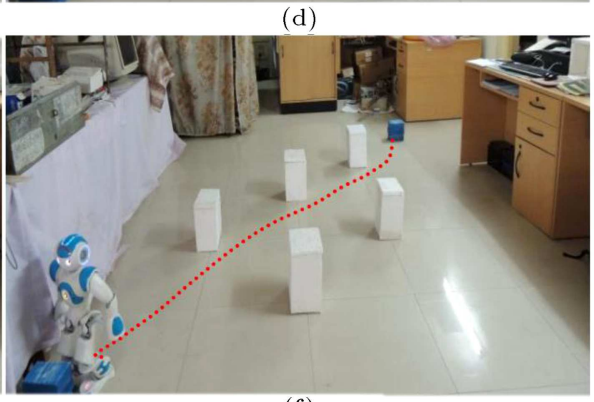

(f)

Figure 10. Experimental result for the navigation of single NAO using RA navigation controller.

Table 3. Comparison between simulation and experimental results for path travelled by a single NAO using RA navigation controller.

\begin{tabular}{cccc}
\hline $\begin{array}{c}\text { No. of } \\
\text { scenario }\end{array}$ & $\begin{array}{c}\text { Path length covered } \\
\text { during simulation } \\
(\mathbf{c m})\end{array}$ & $\begin{array}{c}\text { Path length covered } \\
\text { during the experiment } \\
(\mathbf{c m})\end{array}$ & $\begin{array}{c}\text { Error } \\
\mathbf{( \% )}\end{array}$ \\
\hline 1 & 269.88 & 286.2 & 5.70 \\
2 & 380.53 & 404.9 & 6.02 \\
3 & 337.39 & 360.3 & 6.36 \\
\hline
\end{tabular}

Table 4. Comparison between simulation and experimental results for time taken by a single NAO using RA navigation controller.

\begin{tabular}{cccc}
\hline $\begin{array}{c}\text { No. of } \\
\text { scenario }\end{array}$ & $\begin{array}{c}\text { Time taken } \\
\text { during simulation } \\
(\mathbf{s e c})\end{array}$ & $\begin{array}{c}\text { Time taken } \\
\text { during experiment } \\
(\mathbf{s e c})\end{array}$ & $\begin{array}{c}\text { Error } \\
(\%)\end{array}$ \\
\hline 1 & 33.90 & 36.11 & 6.12 \\
2 & 47.79 & 51.08 & 6.44 \\
3 & 42.45 & 45.58 & 6.86 \\
\hline
\end{tabular}

such as loss in Wi-Fi data transmission, the presence of friction, slipping effects at the contact point between the foot of the humanoid and floor, etc. These factors increase the navigational parameters to some extent. After recording both the simulated and experimental results, the percentage of errors were calculated. It was observed that, in all cases, the error percentage was around $7 \%$, which is well below the acceptable limit.

\section{Navigation of multiple humanoids in a complex environment}

Path planning and navigation of multiple humanoids can be excessively challenging than the navigation of a single humanoid. The reason behind the above case is that, in a single humanoid problem, the environment is static, and when multiple humanoids navigate, it 
becomes a dynamic one. In a dynamic environment, each humanoid has to avoid the static obstacles that are present in the path and the dynamic fellow humanoids, which are navigating, simultaneously, in the same platform.

\subsection{Simulation and experiment in dynamic environment}

For the simulation of multiple humanoids, V-REP was again selected as the simulation software. In the current work of navigation of multiple humanoid robots, three humanoid NAOs have been considered in a single environment. The environment size is kept exactly the same as that for static environment analysis. Four static obstacles are considered in the analysis at random positions. Each humanoid has its own pre-defined source and goal position. It has to be kept in mind that the rules of regression navigational controller can avoid the obstacles, but not decide anything regarding the priorities if a conflicting situation arises. Therefore, along with the regression navigational control, the logic of the Petri-net controller is also considered in the current problem. The working pattern of the Petri-net controller has been already described in the previous sections. The environment size for navigation of multiple humanoids is kept as $200 \times 250$ units with four numbers of static obstacles. Along with the static obstacles, each humanoid acts as a dynamic obstacle to the other two. The three humanoid NAOs (denoted as N1, N2, and N3) have their predefined sources or start positions (denoted as S1, S2, and S3) and goals or target positions (T1, T2, and T3). A program has been written in the LUA language using the combined logic and rules of the regression navigational controller and Petri-net controller; it has been implemented in all the humanoids. After the setting of the environment, the three humanoids started their journey to reach their respective goal positions. Figure 11 illustrates the simulation environmental setup for the navigation of multiple humanoids and the navigation of each humanoid to their respective goals.

It can be observed from Figure 11(a) that each humanoid has been marked with their start and goal positions. Then, they started their journey to their respective goals. Based on Figure 11(a) to (g), all the humanoids have avoided both the static and dynamic obstacles and reached their targets safely.

To validate the results of simulation analysis, a practical experimental setup was developed in the laboratory conditions as that in the case of navigation of a single humanoid robot. The platform size was chosen as $200 \times 250 \mathrm{~cm}$. Four numbers of static obstacles were set at similar positions. A program was written by using the logic of regression navigational control and Petri-net controller and implemented in all the humanoid NAOs. After the practical platform was ready, all the humanoids started their journey towards their respective targets. Figure 12 represents the actual setup that has been used for the experiments and the navigational pattern followed by the multiple humanoid robots.

Based on Figure 12(a), all the three humanoids are marked with their corresponding start and goal positions. After the start signal, all of them moved forward towards their respective goal positions. Based on Figure 12(a) to (h), all the humanoids have avoided the static obstacles and the inter-collision between them. The navigational parameters, such as path length and time taken, are measured in a similar way as were done in the case of a single humanoid robot, i.e., by measuring tape and stopwatch, respectively. Finally, a comparison was done among the simulated and experimental results, and the data are presented in Tables 5 and 6 . Table 5 represents a comparison of

Table 5. Comparison between simulation and experimental results for path travelled by multiple NAOs using RA navigation controller.

\begin{tabular}{|c|c|c|c|c|c|c|c|c|c|}
\hline \multirow{3}{*}{ Sl. no } & Simu & tion & sults & Expe & ment & esults & \multirow{2}{*}{\multicolumn{3}{|c|}{ Errors (\%) }} \\
\hline & \multicolumn{6}{|c|}{ Path travelled $(\mathrm{cm})$} & & & \\
\hline & N1 & N2 & N3 & N1 & N2 & N3 & N1 & N2 & N3 \\
\hline 1 & 285.5 & 243.5 & 267.7 & 298.6 & 257.6 & 283.7 & 4.39 & 5.47 & 5.64 \\
\hline 2 & 314.1 & 269.8 & 298.6 & 325.8 & 285.5 & 320.2 & 3.59 & 5.5 & 6.74 \\
\hline 3 & 244.7 & 295.6 & 230.9 & 258.9 & 309.8 & 246.7 & 5.48 & 4.58 & 6.4 \\
\hline
\end{tabular}

Table 6. Comparison between simulation and experimental results for time taken by multiple NAOs using RA navigation controller.

\begin{tabular}{|c|c|c|c|c|c|c|c|c|c|}
\hline \multirow{3}{*}{ Sl. no } & \multicolumn{3}{|c|}{ Simulation results } & \multicolumn{3}{|c|}{ Experimental results } & \multirow{2}{*}{\multicolumn{3}{|c|}{ Errors (\%) }} \\
\hline & \multicolumn{6}{|c|}{ Time required $(\mathrm{sec})$} & & & \\
\hline & N1 & N2 & N3 & N1 & N2 & N3 & N1 & N2 & N3 \\
\hline 1 & 35.67 & 29.43 & 32.5 & 38.1 & 31.43 & 34.5 & 6.38 & 6.36 & 5.8 \\
\hline 2 & 39.54 & 34.67 & 36.12 & 41.6 & 36.9 & 38.6 & 4.95 & 6.04 & 6.42 \\
\hline 3 & 29.71 & 25.19 & 27.95 & 31.3 & 26.8 & 29.5 & 5.08 & 6.01 & 5.25 \\
\hline
\end{tabular}




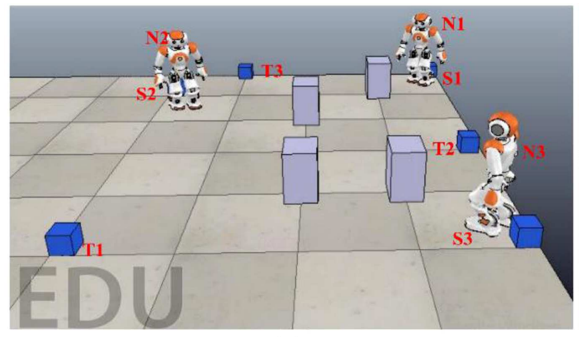

(a)

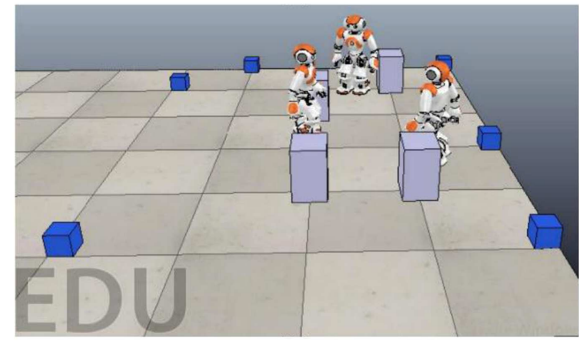

(c)

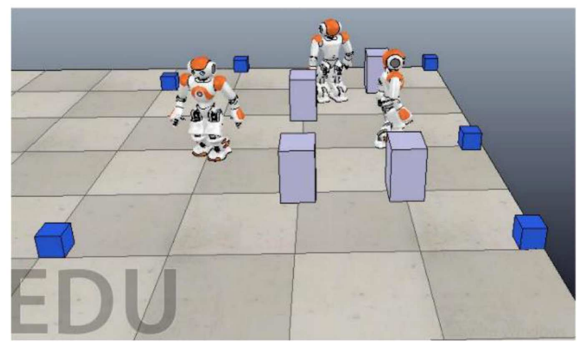

(e)

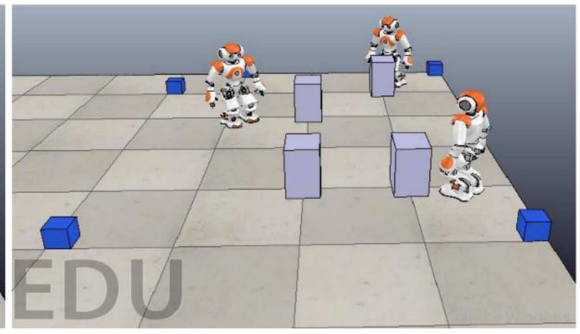

(b)

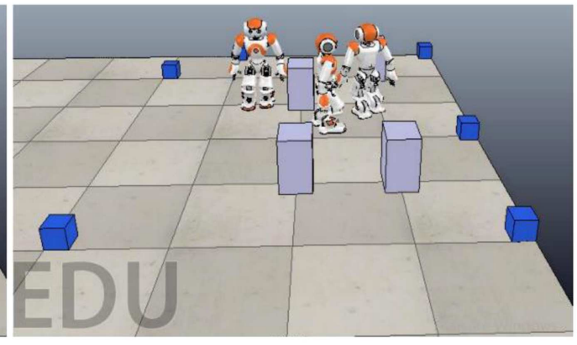

(d)

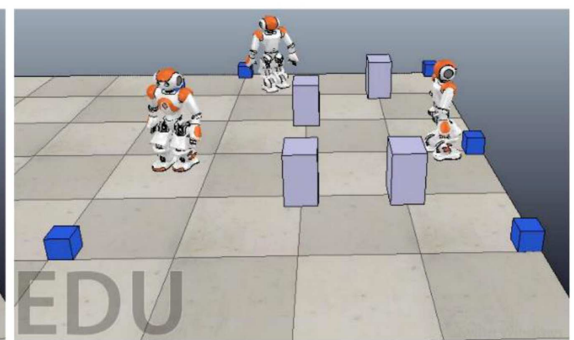

(f)

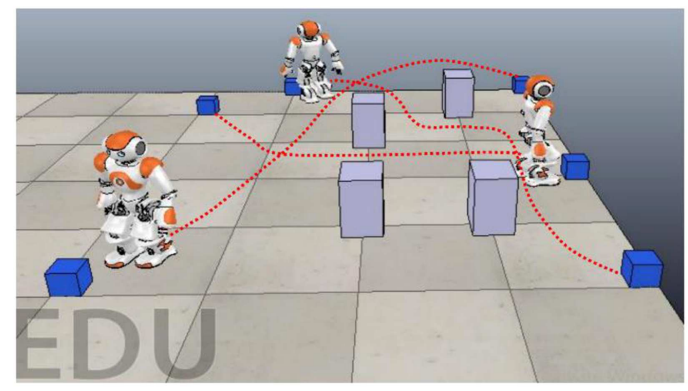

(g)

Figure 11. Simulation result for the navigation of multiple NAOs using RA navigation controller and Petri-net model.

path length between the simulated and experimental environments, and Table 6 represents the comparison for time taken between simulated and experimental environments.

It can be observed that the navigational parameters show a higher value in experimental results than that in the simulation results. The percentage of errors for all the comparisons is around $7 \%$, which is well under the acceptable limit.

\section{Comparison of the proposed regression navigational controller with other existing techniques}

From the above sections, it was observed that the proposed regression navigation controller is successfully implemented in both single and multiple humanoid robots. The humanoids were perfectly able to avoid both static and dynamic obstacles and reach their goal positions safely. However, to have a detailed investigation regarding the efficiency of the proposed navigation controller, it is required to compare it with other existing techniques. To do so, a Co-Evolutionary improved Genetic Algorithm (CEGA) and an improved Genetic Algorithm (IGA) are chosen. CEGA and IGA are heuristic methods as compared to the regression analysis, which is a statistical method. CEGA and IGA work on the basis of a predefined objective function, while regression analysis is based on statistical formula and training data. Qu et al. [42] developed two 


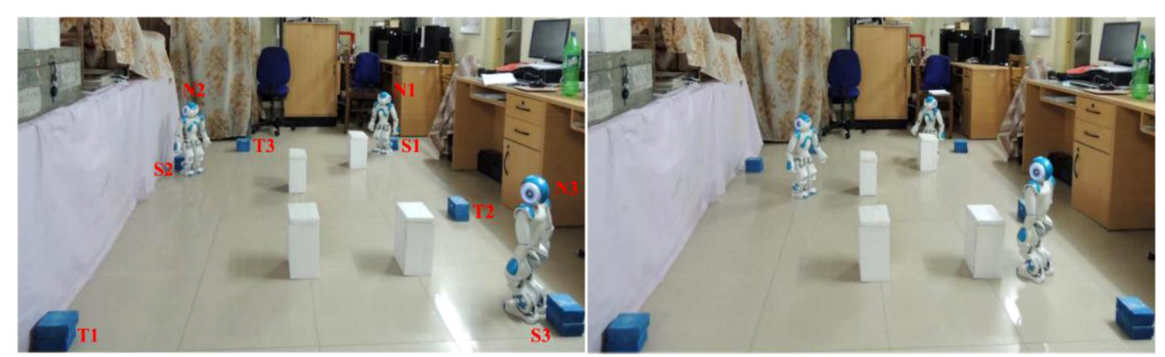

(a)

(b)

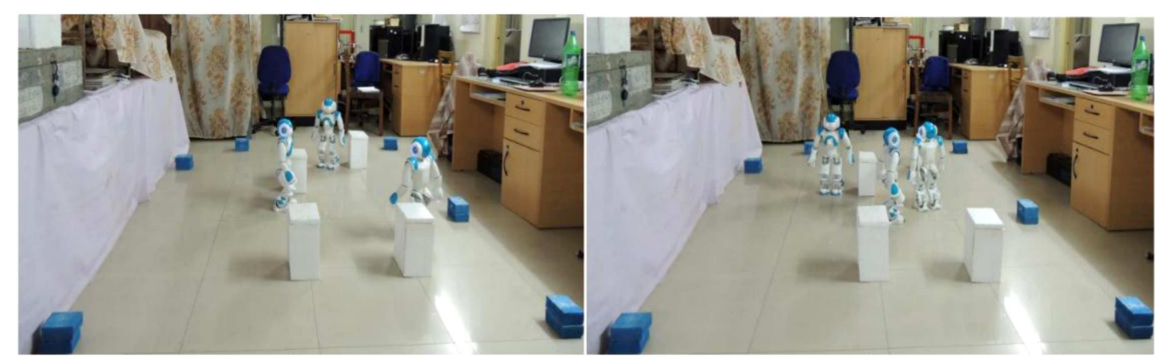

(c)

(d)

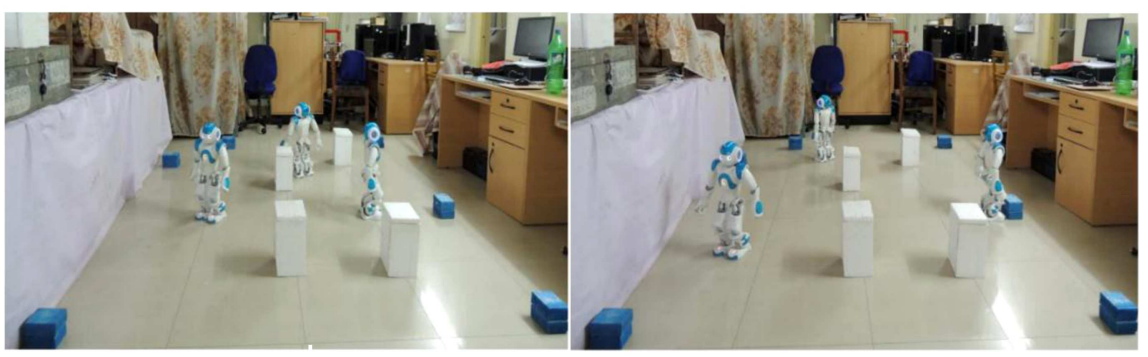

(e)

(f)

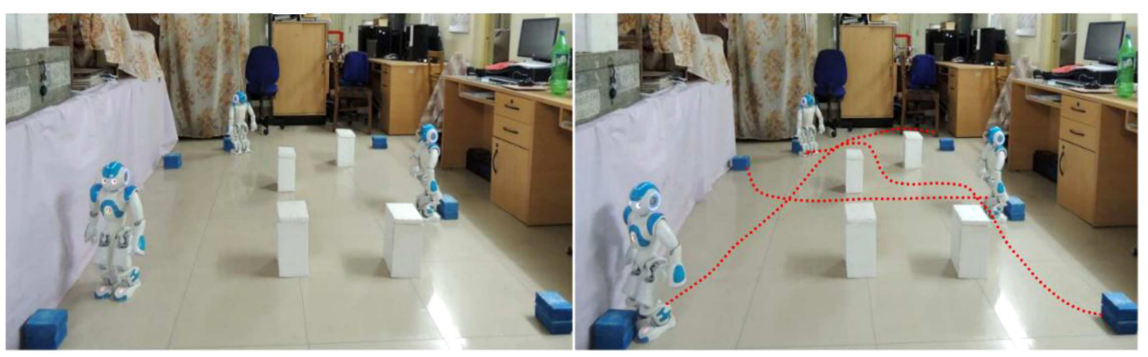

(g)

(h)

Figure 12. Experimental result for the navigation of multiple NAOs using RA navigation controller and Petri-net model.

methods, named as Improved Genetic Algorithm and CEGA. In the current analysis, navigation of single and multiple robots was compared with IGA and CEGA algorithms, respectively. Figures 13 and 14 demonstrate a comparison between IGA and proposed technique to navigate a single robot.

Figures 15 and 16 demonstrate the comparison between CEGA and the proposed navigation controller to navigate multiple robots.

The navigational parameters, such as the path length and time taken, are calculated for the respective existing and proposed techniques, and a comparison is done between them. Tables 7 and 8 represent the comparison for path length between the existing and proposed methods for the navigation of single and multiple robots, respectively.

From the obtained results, it is quite evident that the proposed navigation controller has served as a better alternative than the existing techniques. Hence, the efficiency of the proposed regression navigational controller is on an enhanced mode than the existing methods.

\section{Conclusions}

Robots have become a non-separable part of human life as they contribute to all the sectors where human efforts are concerned. With an increasing demand of 
humanoid robots in several sectors such as automobile, manufacturing, medical assistance, etc., the path planning and navigation of humanoid robots in their respective workspace have emerged as a challenging area in the robotics research. Considering the above objective, the current investigation was aimed at designing a regression navigational controller that can navigate the humanoid robot both in static and dynamic environ-

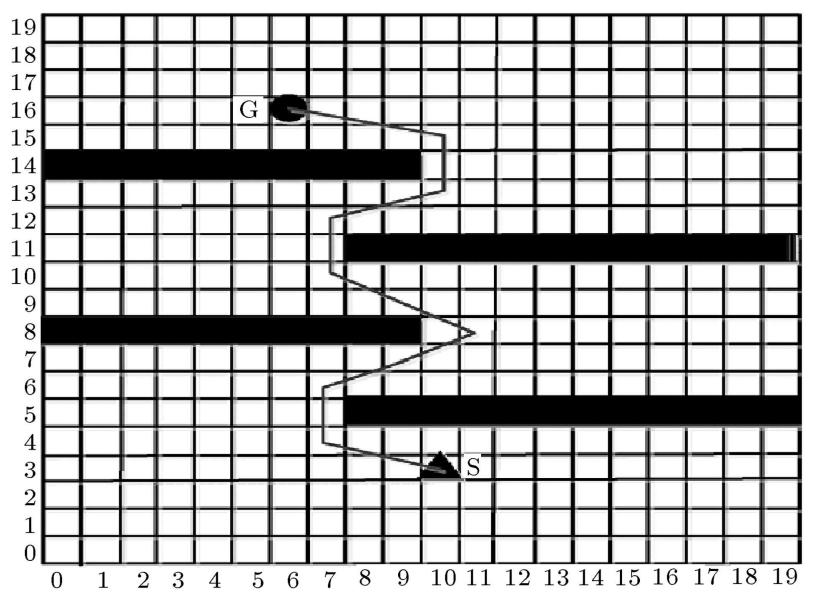

Figure 13. Simulation result for a single robot using IGA algorithm [42].

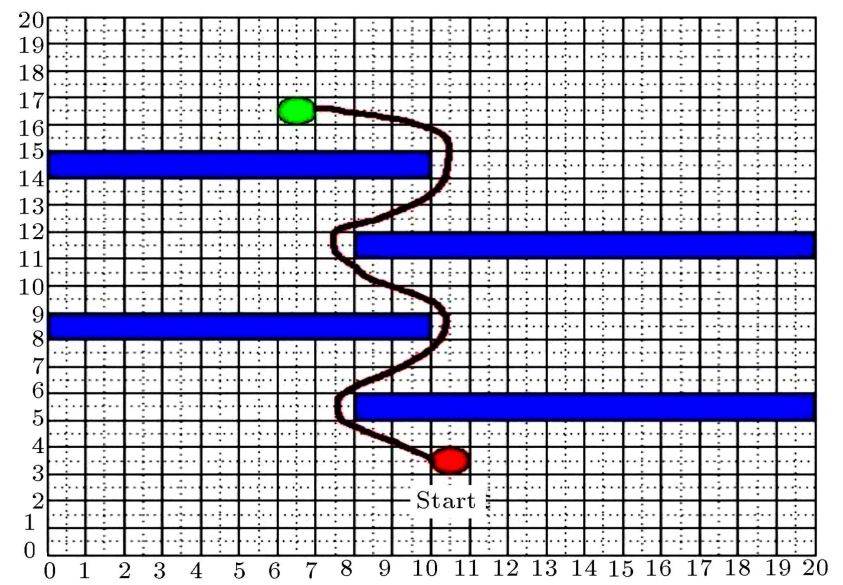

Figure 14. Simulation result for a single robot using RA navigation controller. ments. As humanoid path planning is very new in its own kind, regression technique was chosen as the path planning approach to humanoid NAO. To enable the multiple humanoids to navigate in a dynamic

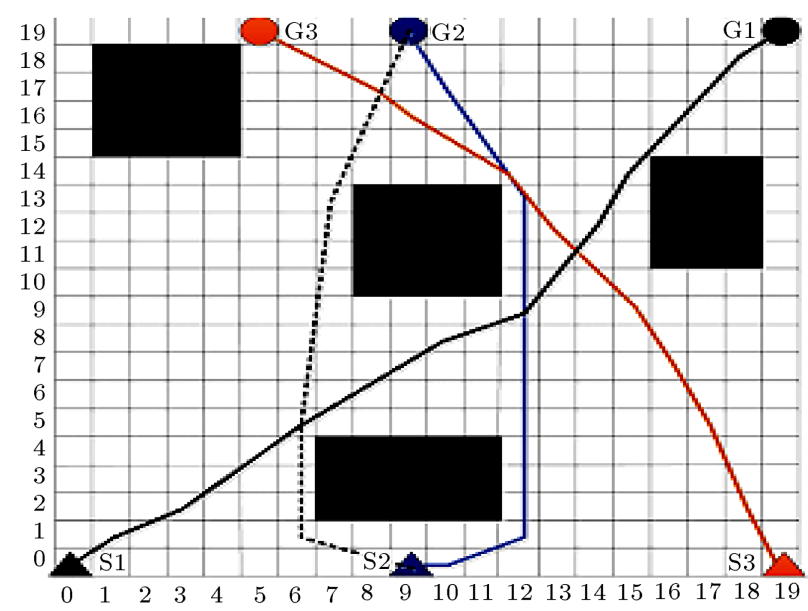

Figure 15. Simulation result for multiple robots using CEGA approach [42].

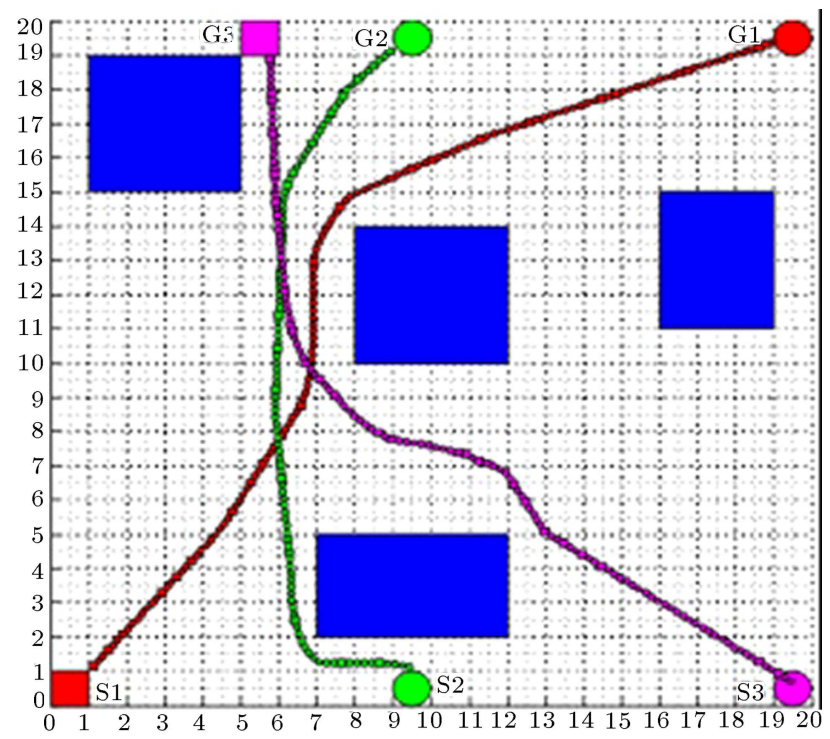

Figure 16. Simulation result for multiple robots using RA navigation controller.

Table 7. Comparison of the results obtained from [42] and proposed RA navigation controller for a single robot navigation.

\begin{tabular}{lcc}
\hline \multicolumn{1}{c}{ Technique used } & Path length $\mathbf{( c m )}$ & Deviation (\%) \\
\hline Qu et al. [42] (Figure 13) & 25.89 & \multirow{2}{*}{5.83} \\
Proposed RA technique (Figure 14) & 24.38 & \\
\hline
\end{tabular}

Table 8. Comparison of the results obtained from [30] and the proposed regression navigation controller for multiple robots navigation.

\begin{tabular}{lcc}
\hline \multicolumn{1}{c}{ Technique used } & Path length $\mathbf{( c m )}$ & Deviation (\%) \\
\hline Qu et al. [42] (Figure 15) & 28.19 & 5.77 \\
Proposed RA Technique(Figure 16) & 26.65 & \\
\hline
\end{tabular}


environment, a Petri-net controller was designed to avoid the inter-collision among the humanoids. Based on the logic and rules of regression analysis and Petrnet controller, the humanoid NAOs were tested for both simulated and experimental environments. A comparison between the results of simulated and experimental environments revealed that all the humanoids were successful in avoiding the obstacles present in their path and reaching their respective goal positions safely.

The workplace where a humanoid is supposed to work is full of uncertainties when dealing with both static and dynamic obstacles. The use of a navigation controller was tested in both static and dynamic environments, leading to satisfactory results. To validate the efficiency of the proposed navigation controller, it was also compared with the existing techniques as available in the literature. The comparison showed that the proposed navigation controller serves as a better technique than the existing ones. Robotic research is the brightest future of the developing science and technology. The path planning and navigation of the humanoid robots would definitely add an entire new dimension to all aspects of robotic technology in its advanced form.

\section{References}

1. Atkinson, A.C. "Robust and diagnostic regression analyses", Communications in Statistics-Theory and Methods, 11(22), pp. 2559-2571 (1982).

2. Asano, T., Asano, T., Guibas, L., Hershberger, J., and Imai, H. "Visibility-polygon search and euclidean shortest paths", 26th Annual Symposium on Foundations of Computer Science, pp. 155-164 (1985).

3. Takahashi, O. and Schilling, R.J. "Motion planning in a plane using generalized Voronoi diagrams", IEEE Transactions on Robotics and Automation, 5(2), pp. 143-150 (1989).

4. Hwang, Y.K. and Ahuja, N. "A potential field approach to path planning", IEEE Transactions on Robotics and Automation, 8(1), pp. 23-32 (1992).

5. Lazaro, J.L., Gardel, A., Mataix, C., Rodriguez, F.J., and Martin, E. "Adaptive workspace modeling, using regression methods, and path planning to the alternative guide of mobile robots in environments with obstacles", 7th IEEE International Conference on Emerging Technologies and Factory Automation, 1, pp. 529-534 (1999).

6. Bai, S. and Low, K.H. "Terrain evaluation and its application to path planning for walking machines", Advanced Robotics, 15(7), pp. 729-748 (2001).

7. Lee, Y.J. and Bien, Z. "Path planning for a quadruped robot: an artificial field approach", Advanced Robotics, 16(7), pp. 609-627 (2002).
8. Minguez, J. and Montano, L. "Nearness diagram (ND) navigation: collision avoidance in troublesome scenarios", IEEE Transactions on Robotics and Automation, 20(1), pp. 45-59 (2004).

9. Benamati, L., Cosma, C., and Fiorini, P. "Path planning using flat potential field approach", 12th International Conference on Advanced Robotics, pp. 103-108 (2005).

10. Liang, T.C., Liu, J.S., Hung, G.T., and Chang, Y.Z. "Practical and flexible path planning for car-like mobile robot using maximal-curvature cubic spiral", Robotics and Autonomous Systems, 52(4), pp. 312-335 (2004).

11. Papadopoulos, E., Papadimitriou, I., and Poulakakis, I. "Polynomial-based obstacle avoidance techniques for nonholonomic mobile manipulator systems", Robotics and Autonomous Systems, 51(4), pp. 229-247 (2005).

12. Masehian, E. and Sedighizadeh, D. "Classic and heuristic approaches in robot motion planning-a chronological review", World Academy of Science, Engineering and Technology, 23, pp. 101-106 (2007).

13. Qi, N., Ma, B., Liu, X.E., Zhang, Z., and Ren, D. "A modified artificial potential field algorithm for mobile robot path planning", 7th World Congress on Intelligent Control and Automation, pp. 2603-2607 (2008).

14. Jolly, K.G., Kumar, R.S., and Vijayakumar, R. "A Bezier curve based path planning in a multi-agent robot soccer system without violating the acceleration limits", Robotics and Autonomous Systems, 57(1), pp. 23-33 (2009).

15. Keshmiri, S. and Payandeh, S. "Multi-robots, multilocations recharging paradigm: a regression route technique", In Proceedings of the 14th IASTED International Conference, Robotics and Applications, Cambridge, MA, USA, pp. 160-165 (2009).

16. Shi, P. and Zhao, Y. "Global path planning for mobile robot based on improved artificial potential function", IEEE International Conference on Automation and Logistics, pp. 1900-1904 (2009).

17. Singh, M.K., Parhi, D.R., and Pothal, J.K. "ANFIS approach for navigation of mobile robots", International Conference on Advances in Recent Technologies in Communication and Computing, pp. 727-731 (2009).

18. Parhi, D.R. and Singh, M.K. "Navigational strategies of mobile robots: a review", International Journal of Automation and Control, 3(2-3), pp. 114-134 (2009).

19. Singh, M.K. and Parhi, D.R. "Path optimisation of a mobile robot using an artificial neural network controller", International Journal of Systems Science, 42(1), pp. 107-120 (2011).

20. Razzazi, M. and Sepahvand, A. "Time complexity of two disjoint simple paths", Scientia Iranica, 24(3), pp. 1335-1343 (2017). 
21. Mohammadi, E., Zohoor, H., and Khadem, S.M. "Design and prototype of an active assistive exoskeletal robot for rehabilitation of elbow and wrist", Scientia Iranica. Transactions B, Mechanical Engineering, 23(3), p. 998 (2016).

22. Kala, R., Shukla, A., and Tiwari, R. "Dynamic environment robot path planning using hierarchical evolutionary algorithms", Cybernetics and Systems: An International Journal, 41(6), pp. 435-454 (2010).

23. Kashmiri, S. and Payandeh, S. "Robot navigation controller: a non-parametric regression approach", IFAC Proceedings, 43(22), pp. 22-27 (2010).

24. Sheng, J., He, G., Guo, W., and Li, J. "An improved artificial potential field algorithm for virtual human path planning", Entertainment for Education. Digital Techniques and Systems, pp. 592-601 (2010).

25. Hong, Z., Liu, Y., Zhongguo, G., and Yi, C. "The dynamic path planning research for mobile robot based on artificial potential field", International Conference on Consumer Electronics, Communications and Networks, pp. 2736-2739 (2011).

26. Mohanty, P.K. and Parhi, D.R. "Optimal path planning for a mobile robot using cuckoo search algorithm", Journal of Experimental \& Theoretical Artificial Intelligence, 28(1-2), pp. 35-52 (2016).

27. Mohanty, P.K. and Parhi, D.R. "Path planning strategy for mobile robot navigation using MANFIS controller", In Proceedings of the International Conference on Frontiers of Intelligent Computing: Theory and Applications, pp. 353-361 (2014).

28. Parhi, D.R. and Mohanty, P.K. "IWO-based adaptive neuro-fuzzy controller for mobile robot navigation in cluttered environments", The International Journal of Advanced Manufacturing Technology, 83(9-12), pp. 1607-1625 (2016).

29. Pham, D.T. and Parhi, D.R. "Navigation of multiple mobile robots using a neural network and a Petri Net model", Robotica, 21(1), pp. 79-93 (2003).

30. Chen, C.Y. and Ko, C.C. "An evolutionary method to vision-based self-localization for soccer robots", Scientia Iranica. Transactions B, Mechanical Engineering, 22(6), p. 2071 (2015).

31. Korayem, M.H., Maddah, S.M., Taherifar, M., and Tourajizadeh, H. "Design and programming a 3D simulator and controlling graphical user interface of ICaSbot, a cable suspended robot", Scientia Iranica. Transactions B, Mechanical Engineering, 21(3), p. 663 (2014).

32. Sayyaadi, H. and Babaee, M. "Control of nonholonomic mobile manipulators for cooperative object transportation", Scientia Iranica. Transaction B, Mechanical Engineering, 21(2), p. 347 (2014).

33. Ohki, T., Nagatani, K., and Yoshida, K. "Local path planner for mobile robot in dynamic environment based on distance time transform method", Advanced Robotics, 26(14), pp. 1623-1647 (2012).
34. Li, G., Yamashita, A., Asama, H., and Tamura, Y. "An efficient improved artificial potential field based regression search method for robot path planning", International Conference on Mechatronics and Automation, pp. 1227-1232 (2012).

35. Hong, J. and Park, K. "A new mobile robot navigation using a turning point searching algorithm with the consideration of obstacle avoidance", The International Journal of Advanced Manufacturing Technology, 52(5), pp. 763-775 (2011).

36. Keshmiri, S. and Payandeh, S. "Regression analysis of multi-rendezvous recharging route in multi-robot environment", International Journal of Social Robotics, 4(1), pp. 15-27 (2012).

37. Tingbin, C. and Qisong, Z. "Robot motion planning based on improved artificial potential field", 3rd International Conference on Computer Science and Network Technology, pp. 1208-1211 (2013).

38. Clever, D. and Mombaur, K.D. "An inverse optimal control approach for the transfer of human walking motions in constrained environment to humanoid robots", In Robotics: Science and Systems (2016).

39. Mirjalili, R., Yousefi-koma, A., Shirazi, F.A., and Mansouri, S. "Online path planning for SURENA III humanoid robot using model predictive control scheme", 4th International Conference on Robotics and Mechatronics, pp. 416-421 (2016).

40. Kofinas, N., Orfanoudakis, E., and Lagoudakis, M.G. "Complete analytical inverse kinematics for NAO", 13th International Conference on Autonomous Robot Systems, pp. 1-6 (2013).

41. Peterson, J.L., Petri Net Theory and the Modeling of Systems, Prentice-Hall, Englewood cliffs (1981).

42. Qu, H., Xing, K., and Alexander, T. "An improved genetic algorithm with co-evolutionary strategy for global path planning of multiple mobile robots", $\mathrm{Neu}$ rocomputing, 120, pp. 509-517 (2013).

\section{Biographies}

Priyadarshi Biplab Kumar completed his BTech from GITA, Bhubaneswar in 2014. After completion of his Bachelor's degree, he joined as a PhD Research Scholar at Mechanical Engineering Department of National Institute of Technology, Rourkela, Odisha, India. He is currently working in the development of navigation controllers for path planning of humanoid robots. His research interests include human robot correlation, kinematics and dynamics of robots and artificial intelligent techniques for navigational purpose.

Chinmaya Sahu is presently working as a PhD Research Scholar at Mechanical Engineering Department of National Institute of Technology, Rourkela, Odisha, India after completing post graduate studies from the same institute. His research areas include humanoid 
robotics, path planning and navigation, and artificial intelligent techniques.

Dayal R. Parhi received his PhD in Mobile Robotics from Cardiff School of Engineering, UK. He has more than 25 years of research and teaching experience. Presently, he is working as a Professor at Mechanical Engineering Department of National Institute of Technology Rourkela. His research interests include humanoid robots, human robot correlation, mobile robot navigation, and crack detection of structures using artificial intelligent techniques.

Krishna Kant Pandey is presently working as a PhD Research Scholar at Mechanical Engineer- ing Department of National Institute of Technology, Rourkela, Odisha, India after completing postgraduate studies from the same institute. His research areas include application of computational intelligence in mobile robots and navigation of different forms of robots.

Animesh Chhotray is presently working as a $\mathrm{PhD}$ Research Scholar at Mechanical Engineering Department of National Institute of Technology, Rourkela, Odisha, India after completing his post-graduate studies from Centre for Mechanics, BPUT. His research areas include stability and balancing of two wheeled mobile robots and use of intelligent algorithms for navigation and path planning. 